Effects of i on si ze and val ence on i on di st $r i$ but $i$ on i $n$ mixed counteri on systens of rodl i ke pol yel ectrol yte sol ut i on. I. Mxed- si ze count eri on systens wi th same val ence

\begin{tabular}{|l|l|}
\hline 著者 & 西尾 卓広, M nakat a Aki ra \\
\hline $\begin{array}{l}\text { j our nal or } \\
\text { publ i cat i on t i t l e }\end{array}$ & J. Chem Phys. \\
\hline vol une & 113 \\
\hline page range & $10784-10792$ \\
\hline year & $2000-12-15$ \\
\hline URL & ht t p: //hdl . handl e. net /10271/2069 \\
\hline
\end{tabular}




\title{
Effects of ion size and valence on ion distribution in mixed counterion systems of rodlike polyelectrolyte solution. I. Mixed-size counterion systems with same valence
}

\author{
Takuhiro Nishio and Akira Minakata \\ Department of Physics, Hamamatsu University School of Medicine, Hamamatsu 431-3192, Japan
}

(Received 13 June 2000; accepted 19 September 2000)

\begin{abstract}
The influence of the counterion size is investigated in the mixed counterion systems of the salt-free polyelectrolyte solution in a cylindrical cell model. The mixtures of two species of the counterion having the same valence and different size are simulated systematically by means of the Monte Carlo method in the primitive model of the rodlike polyelectrolyte solution. The results of the free fractions and the selectivity coefficients are compared with the numerical solutions of the PoissonBoltzmann equation. The observed differences between both methods are explained in terms of the ion-ion correlations and the effect of finite ion size. (C) 2000 American Institute of Physics. [S0021-9606(00)50147-0]
\end{abstract}

\section{INTRODUCTION}

In the study of polyelectrolyte solution, the size and valence of counterions are notable parameters. The thermodynamic properties of the solution and the ion distributions are dependent on the counterion species. The selectivity of the counterions by the polyion, which is widely observed in the biomolecular systems, seems to be due primarily to the difference in the ion size and valence. Then, this selectivity can be analyzed as a consequence of the polyelectrolyte research. In particular, the mixed counterion systems with different size and/or valence are worth investigating quantitatively. Their competition with the charged site of the polyion is important to understand the characteristics of the polyionsmall ion interactions.

The effects of the ion size and valence can be interpreted essentially by the simple two-phase approximation to the linear polyelectrolyte solutions. ${ }^{1}$ One of the practical methods to investigate the ion distribution quantitatively is solving the nonlinear Poisson-Boltzmann (PB) equation for the cell model with cylindrical symmetry. ${ }^{2-6}$ However, the deficiencies inherent in the PB approach have been recognized such as the neglects of the ion-ion correlations and of the effect of finite ion size. ${ }^{7}$ To overcome these deficiencies, improvements have been attempted on the basis of the other theoretical frameworks. ${ }^{8,9}$ The Monte Carlo (MC) simulation is another preferable way to remove the approximations in the theories. Throughout several studies, the discrepancy between $\mathrm{MC}$ result and PB solution has been recognized. ${ }^{10-12}$ The MC method has the advantage of the consideration of the behavior of individual ions even in a primitive model. Recently, the refinements in the simulations were extensively attempted for the DNA molecular model. ${ }^{13}$ The competitive bindings of counterions to DNA were also investigated by the simulations. ${ }^{14-17}$

The aim of the present study is to examine the influences of ion size and valence through the canonical MC simulations for the mixed counterion systems of the rodlike polyelectrolyte solution generally. The systematic comparison of the MC results with the PB solutions must provide a contri. bution to the fundamental knowledge of the polyion-small ion interaction. The results of the mixtures of two counterion species with the same valence and different size are repre sented in this manuscript. The mixed-valence systems will be reported in the following paper.

The cases of no added salt are presented here to clanify their influences on the thermodynamic properties of the solution. In order to extract the elementary features in the mixed ion system, a polyion is assumed to be a simple rod with uniformly smeared charge in the continuum dielectric model. Analytical expression of the PB solution in the salt: free case was given for the system of mixed counterions with different size, and the preferential selectivity by the polyion for the smaller counterion was presented. ${ }^{18,19}$ In the present study, numerical analysis for the differential equation is ut. lized to solve the PB equations. This method provides an alternative way to evaluate the ion distributions more easily, and to be applicable to the mixed-valence system.

In the MC system corresponding to the PB system, the primitive model is adopted. The mobile counterions at? treated as finite-sized spheres with hard core potential. The radial counterion distributions are evaluated around the poly. ion in a cylindrical cell at various sets of parameters in the MC simulations with high accuracy. Some discussion is given for the dependence of the ion distribution and selectiv ity on each parameter and observed differences between the MC and PB methods.

\section{METHOD}

\section{A. Solution of the Poisson-Boltzmann equation}

Analytical and numerical procedures have been pro sented for the solutions of the PB equation in mixed courterion systems in cylindrical cell model under varios conditions. ${ }^{18-21}$ In this study, an accurate numerical solution is achieved by applying the initial value problem of the one 
dimensional ordinary differential equation to the boundary value problem. The general procedure is explained below.

In an infinitely long cylindrical cell whose radius is $R$, an impenetrable polymer rod whose radius is $a$, is assumed at the center of the cell. The mixture of two species (A and B) of spherical mobile counterions with different size and/or valence is considered in the continuum dielectric model. The negative charge is assumed to be smeared uniformly on the surface of the polymer cylinder. The value, $b$, is defined as a mean distance of an elementary charge along the polymer axis. The radii and positive valences of the counterion $\mathrm{A}$ and $\mathrm{B}$ are defined as $\sigma_{\mathrm{A}}, \sigma_{\mathrm{B}}$, and $z_{\mathrm{A}}$, and $z_{\mathrm{B}}$, respectively. When the radius of the ion $\mathrm{A}$ is different from that of ion $\mathrm{B}$, the zone for the equation is divided into two regions (inner region and outer region) for the radial distance from the center of the cell, $r$, in consideration of the distance of closest approach of each ion species. The definitions are adopted such that $y=e \psi / k_{\mathrm{B}} T$ is the reduced electrostatic potential and $l_{\mathrm{B}}=e^{2} /\left(4 \pi \varepsilon_{0} D k_{\mathrm{B}} T\right)$ is the Bjerrum length, where $e$ is the elementary protonic charge, $\psi$, the electrostatic potential, $k_{\mathrm{B}}$, the Boltzmann constant, $T$, the absolute temperature, $\varepsilon_{0}$, he permittivity of the vacuum, and $D$, the relative dielectric constant of the solvent water. In the case that the radius of the ion $\mathrm{A}$ is smaller than that of ion $\mathrm{B}\left(\sigma_{\mathrm{A}}<\sigma_{\mathrm{B}}\right)$, the PB equations without added salt are expressed in the SI (Système International) as

$$
\begin{aligned}
& \frac{1}{r} \frac{d}{d r}\left(r \frac{d y_{i}}{d r}\right)=-4000 \pi l_{\mathrm{B}} N_{\mathrm{AV}}\left[z_{\mathrm{A}} C_{\mathrm{A} 0} \exp \left(-z_{\mathrm{A}} y_{i}\right)\right] \\
& a+\sigma_{\mathrm{A}} \leqslant r \leqslant a+\sigma_{\mathrm{B}} \\
& \frac{1}{r} \frac{d}{d r}\left(r \frac{d y_{o}}{d r}\right)=-4000 \pi l_{\mathrm{B}} N_{\mathrm{AV}}\left[z_{\mathrm{A}} C_{\mathrm{A} 0} \exp \left(-z_{\mathrm{A}} y_{o}\right)\right. \\
& \left.+z_{\mathrm{B}} C_{\mathrm{B} 0} \exp \left(-z_{\mathrm{B}} y_{o}\right)\right] \\
& a+\sigma_{\mathrm{B}} \leqslant r \leqslant R,
\end{aligned}
$$

where $N_{\mathrm{AV}}$ is Avogadro's constant, and $C_{\mathrm{A} 0}$ and $C_{\mathrm{B} 0}$ are the molar concentration of the counterion $\mathrm{A}$ and $\mathrm{B}$ at the radius where the electrostatic potential is equal to zero, respectively. The reduced electrostatic potentials in inner and outer regions are marked by the subscript $i$ and $o$, respectively. The unit of molar concentration $C$ is $\mathrm{mol} / \mathrm{dm}^{3}$. The boundary conditions are described in the polyanion solution as

$$
\begin{aligned}
& \left(\frac{d y_{i}}{d r}\right)_{r=a+\sigma_{\mathrm{A}}}=\frac{2 \xi}{a+\sigma_{\mathrm{A}}}, \quad\left(\frac{d y_{o}}{d r}\right)_{r=R}=0, \\
& y_{i}\left(a+\sigma_{\mathrm{B}}\right)=y_{o}\left(a+\sigma_{\mathrm{B}}\right), \\
& \left(\frac{d y_{i}}{d r}\right)_{r=a+\sigma_{\mathrm{B}}}=\left(\frac{d y_{o}}{d r}\right)_{r=a+\sigma_{\mathrm{B}}},
\end{aligned}
$$

where $\xi=e^{2} /\left(4 \pi \varepsilon_{0} D b k_{\mathrm{B}} T\right)=l_{\mathrm{B}} / b$ is the charge density parameter. ${ }^{22}$ The integrations of the ion concentration are defined as

$$
2 \int_{a+\sigma_{\mathrm{A}}}^{R} C_{\mathrm{A} 0} \exp \left(-z_{\mathrm{A}} y\right) r d r / R^{2}=\bar{C}_{\mathrm{A}},
$$

$$
2 \int_{a+\sigma_{\mathrm{B}}}^{R} C_{\mathrm{B} 0} \exp \left(-z_{\mathrm{B}} y\right) r d r / R^{2}=\bar{C}_{\mathrm{B}},
$$

where $\bar{C}_{\mathrm{A}}$ and $\bar{C}_{\mathrm{B}}$ are the mean molar concentration of the counterion $\mathrm{A}$ and $\mathrm{B}$ in the cell volume, respectively. If the equivalent molar concentration of the polyion, $C_{P}$, is defined as

$$
C_{P}=\frac{1}{1000 N_{\mathrm{AV}}} \frac{1}{\pi R^{2} b}=\frac{1}{1000 N_{\mathrm{AV}}} \frac{\xi}{\pi R^{2} l_{\mathrm{B}}},
$$

the electroneutrality condition is presented as

$$
C_{P}=z_{\mathrm{A}} \bar{C}_{\mathrm{A}}+z_{\mathrm{B}} \bar{C}_{\mathrm{B}} \text {. }
$$

In the practical calculations, the following definition and translation are utilized: ${ }^{23}$

$$
\begin{aligned}
& \kappa^{2}=8000 \pi l_{\mathrm{B}} N_{\mathrm{AV}} C_{\mathrm{A} 0}, \\
& u=\ln \kappa r .
\end{aligned}
$$

Then, Eqs. (1)-(6) are converted into the following equations:

$$
\begin{aligned}
& \frac{d^{2} y_{i}}{d u^{2}}=-\frac{1}{2} \exp (2 u)\left[z_{\mathrm{A}} \exp \left(-z_{\mathrm{A}} y_{i}\right)\right] \\
& u_{\mathrm{A}} \leqslant u \leqslant \\
& \frac{d^{2} y_{o}}{d u^{2}}=-\frac{1}{2} \exp (2 u)\left[z_{\mathrm{A}} \exp \left(-z_{\mathrm{A}} y_{o}\right)+z_{\mathrm{B}} r_{\mathrm{B}, \mathrm{A}}\right. \\
&\left.\times \exp \left(-z_{\mathrm{B}} y_{o}\right)\right]
\end{aligned}
$$$$
u_{\mathrm{A}} \leqslant u \leqslant u_{\mathrm{B}},
$$$$
u_{\mathrm{B}} \leqslant u \leqslant \ln \kappa R \text {, }
$$

$$
\begin{aligned}
& \left(\frac{d y_{i}}{d u}\right)_{u=u_{\mathrm{A}}}=2 \xi, \quad\left(\frac{d y_{o}}{d u}\right)_{u=\ln \kappa R}=0, \\
& y_{i}\left(u_{\mathrm{B}}\right)=y_{o}\left(u_{\mathrm{B}}\right), \quad\left(\frac{d y_{i}}{d u}\right)_{u=u_{\mathrm{B}}}=\left(\frac{d y_{o}}{d u}\right)_{u=u_{\mathrm{B}}},
\end{aligned}
$$

$2 \int_{u_{\mathrm{A}}}^{\ln \kappa R} C_{\mathrm{A} 0} \exp \left(-z_{\mathrm{A}} y\right) \exp (2 u) d u /(\kappa R)^{2}=\bar{C}_{\mathrm{A}}$,

$$
2 \int_{u_{\mathrm{B}}}^{\ln \kappa R} C_{\mathrm{B} 0} \exp \left(-z_{\mathrm{B}} y\right) \exp (2 u) d u /(\kappa R)^{2}=\bar{C}_{\mathrm{B}},
$$

where $\quad u_{\mathrm{A}}=\ln \kappa\left(a+\sigma_{\mathrm{A}}\right), \quad u_{\mathrm{B}}=\ln \kappa\left(a+\sigma_{\mathrm{B}}\right), \quad$ and $\quad r_{\mathrm{B}, \mathrm{A}}$ $=C_{\mathrm{B} 0} / C_{\mathrm{A} 0}$. In the case of counterions with the same radius, the equation for the inner region is not necessary.

Until the conditions Eqs. (13)-(16) are fulfilled, Eqs. (11) and (12) are solved iteratively varying the value of $y$ at $r=R$ and $C_{\mathrm{A} 0}, C_{\mathrm{B} 0}$ with the fixation $\left(d y_{o} / d u\right)_{u=\ln \kappa R}=0$. The integrations in Eqs. (15) and (16) are evaluated by Simpson's rule. The single shooting method is efficient to reduce the number of iterations. The subroutine program on the basis of the Runge-Kutta-Verner method is applied for the initial value problem of the differential equation. ${ }^{24}$ Error of the electroneutrality below $10^{-6}$, whose definition is given later in the explanation of Eq. (27), is achieved by dividing the zone of $u$ into 1024 or more mesh points. The correctness 
of the output is confirmed by comparison with the analytical solutions. The programs following the procedure are applicable also to the mixed-valence systems, in which the analytical solution is not known.

\section{B. Monte Carlo simulation}

In the cylindrical cell system, which is identical to that of the PB calculation, the canonical MC simulations of the ion distribution are performed. Starting from random distribution of counterions, the MC trial of the ion movement is carried out in the isolated cylindrical cell following the standard MC sampling algorithm. ${ }^{25}$ To assume the polyion cylinder to be infinitely long, the MC cell system is constructed from the central cell whose height is $H$, and two infinitely long external cells in both axial sides. The cell height in the simulation is determined from the polyion concentration and the charge density parameter to avoid cell dimensions that are too oblate. ${ }^{26}$

The interaction between the polyion and counterion are given as $-e^{2} z \ln r /\left(2 \pi \varepsilon_{0} D b\right)$. The energy between two mobile ions, labeled as $i$ and $j$, is defined as the Coulombic interaction with the hard core repulsion, as follows:

$$
u_{i j}=\left\{\begin{array}{l}
\frac{e^{2}}{4 \pi \varepsilon_{0} D} \frac{z_{i} z_{j}}{\left|\mathbf{r}_{i}-\mathbf{r}_{j}\right|} \quad\left|\mathbf{r}_{i}-\mathbf{r}_{j}\right|>\sigma_{i}+\sigma_{j} \\
\infty \quad\left|\mathbf{r}_{i}-\mathbf{r}_{j}\right| \leqslant \sigma_{i}+\sigma_{j},
\end{array}\right.
$$

where $z, \mathbf{r}$, and $\sigma$ with subscript $i$ or $j$ denote the valence, the position, and the radius of each ion, respectively. The contribution of the electrostatic energy from the external cell is also considered to eliminate the end effect of the system. The practical calculation of the electrostatic energy is carried out using the minimum image (MI) energy with a pair of ions in the central cell and the self-consistent method for the external potential. Then, the total electrostatic energy, $U_{\mathrm{el}}$ is represented considering the interaction between the polyion and mobile ions, as follows:

$$
\begin{aligned}
U_{\mathrm{el}}= & \sum_{i} \frac{-e^{2} z_{i}}{2 \pi \varepsilon_{0} D b} \ln r_{i}+\sum_{\langle i j\rangle} \frac{e^{2}}{4 \pi \varepsilon_{0} D} \frac{z_{i} z_{j}}{\left|\mathbf{r}_{i}-\mathbf{r}_{j}\right|_{\mathrm{MI}}} \\
& +\sum_{i} e z_{i} \Phi_{\mathrm{EXT}}^{\infty}\left(\mathbf{r}_{i}\right),
\end{aligned}
$$

where $r_{i}$ is the radial position of ion $i$, and $\Phi_{\mathrm{EXT}}^{\infty}\left(\mathbf{r}_{i}\right)$ is the external potential at position, $\mathbf{r}_{i}$, that is, the contribution of the potential from external cells due to the radial charge distributions, which are given by averaging those in the central cell, extending from $H / 2$ to $\infty .^{27}$

A counterion can be moved to a new trial position expressed by the Cartesian coordinates within a definite step width using uniform pseudorandom numbers. The trial is rejected if the collision occurs with the polymer rod or other ions. The trial is also rejected if the ion moves away from the cylindrical cell. If the new axial position, $z$, is less than zero, it is replaced by $z+H$. If $z$ is greater than $H$, it is replaced by $z-H$. The probability function of the acceptance is described, as follows:

$$
P_{\mathrm{M}}=\min \left\{1, \exp \left[-\Delta U_{\mathrm{el}} / k_{\mathrm{B}} T\right]\right\},
$$

where $\Delta U_{\mathrm{el}}$ is the electrostatic energy change with the movement. The step width of the movement trial is regulated to give the acceptance ratio of roughly 0.5 in the early discarded loop for each ion species. The results are not as affected by the ratio within the range $0.40-0.51$ whenever the adequate numbers of trials is attempted.

The small ion distribution is evaluated by summing up the ions in the coaxial cylindrical shell counters, whose width is $1 \AA$, during a sufficiently long trial loop. In the isolated MC cell system, inhomogeneity in the ion distribution appears near the outer cell boundary in the added salt case. ${ }^{28}$ Nevertheless, this artifact is not detected in the cases of salt-free or low salt. ${ }^{29}$ The number of each counterion in the whole cell volume is in the range from 20 to 200. The iterations (4-48 $\times 10^{5}$ Monte Carlo steps per ion) are carried out until a mean error of the concentration in the outermost shell is below $1 \%$ in almost all cases. As the random number generator, mainly the generalized Fibonacci method and partially the shift register method are applied. ${ }^{30-32}$ Although the qualitative difference depending on the number of iterations or the random number generator is not observed, the slight deviations (several percent) over the statistical error are detected in some simulations in which the free fractions (see below) are very low. Then, the results from longer iteration using the former random number generator are adopted.

\section{Representation of results and parameters}

The dependences of the ion distribution on the linear charge density, $\xi$, hydrated ion radius, $\sigma$, polyion rod radius, $a$, polyion concentration, $C_{P}$, and the fraction of ion mix. ture, $w$ (see below), are investigated in the mixture of monovalent-monovalent and divalent-divalent counterions. In addition, some trials of the trivalent-trivalent ion mixture are examined. The simulation conditions are chosen by tak. ing account of a few points such that (a) dependences on the ion size difference are obtainable; (b) precise MC simulations are feasible; and (c) the conditions are in the range close to the practical experiments.

To represent the accumulation of the ions, the ratios of the ion concentration at the outer cell boundary to the mean concentration are evaluated as

$$
f_{\mathrm{A}}=\frac{C_{\mathrm{A}}(R)}{\bar{C}_{\mathrm{A}}}, \quad f_{\mathrm{B}}=\frac{C_{\mathrm{B}}(R)}{\bar{C}_{\mathrm{B}}} .
$$

The values are equivalent to the single ion activity coefficients in the PB system. ${ }^{5}$ This value is also one of the definitions of the fraction of free counterion. ${ }^{20,33}$ Consequently, this value is tentatively termed " free fraction"' in this manuscript. The low value of the free fraction means that the ions are accumulated strongly near the polyion as revealed by the distribution profile. The selectivity coefficient of ion $\mathrm{A}$ against ion B is given as follows: ${ }^{19}$

$$
K_{\mathrm{A}, \mathrm{B}}=\frac{\bar{C}_{\mathrm{A}}}{\bar{C}_{\mathrm{B}}} \frac{C_{\mathrm{B}}(R)}{C_{\mathrm{A}}(R)}=\frac{f_{\mathrm{B}}}{f_{\mathrm{A}}} .
$$


In the MC simulation, the concentration at the outer cell boundary is calculated from the fitting of ion distribution in the outer region near the boundary $(\sim R / 10)$ by a quadratic function.

In the representation of the ion distribution, the integrated fraction of the ion from inner boundary to position $r$, $q(r)$ is evaluated as

$$
\begin{aligned}
& q_{\mathrm{A}}(r)=\frac{\int_{a+\sigma_{\mathrm{A}}}^{r} C_{\mathrm{A}}\left(r^{\prime}\right) r^{\prime} d r^{\prime}}{\int_{a+\sigma_{\mathrm{A}}}^{R} C_{\mathrm{A}}\left(r^{\prime}\right) r^{\prime} d r^{\prime}}, \\
& q_{\mathrm{B}}(r)=\frac{\int_{a+\sigma_{\mathrm{B}}}^{r} C_{\mathrm{B}}\left(r^{\prime}\right) r^{\prime} d r^{\prime}}{\int_{a+\sigma_{\mathrm{B}}}^{R} C_{\mathrm{B}}\left(r^{\prime}\right) r^{\prime} d r^{\prime}} .
\end{aligned}
$$

The following relation is evaluated in the PB system in the case of the same valence:

$$
q_{\mathrm{A}}\left(a+\sigma_{\mathrm{B}}\right)=1-\frac{1}{K_{\mathrm{A}, \mathrm{B}}}=1-\frac{f_{\mathrm{A}}}{f_{\mathrm{B}}} .
$$

In some examinations, the calculations are executed by varying the fraction of ion composition. Thereby, the equivalent fraction of ion $\mathrm{A}$ is represented as

$$
w_{\mathrm{A}}=\frac{z_{\mathrm{A}} \bar{C}_{\mathrm{A}}}{z_{\mathrm{A}} \bar{C}_{\mathrm{A}}+z_{\mathrm{B}} \bar{C}_{\mathrm{B}}} .
$$

Obviously, the equivalent fraction of ion $\mathrm{B}$ is given as $w_{\mathrm{B}}$ $=1-w_{\mathrm{A}}$. Also, the value $\phi$, equivalent to the osmotic coefficient, is calculated as follows: ${ }^{28}$

$$
\phi=\frac{C_{\mathrm{A}}(R)+C_{\mathrm{B}}(R)}{\bar{C}_{\mathrm{A}}+\bar{C}_{\mathrm{B}}}=\frac{f_{\mathrm{A}} \bar{C}_{\mathrm{A}}+f_{\mathrm{B}} \bar{C}_{\mathrm{B}}}{\bar{C}_{\mathrm{A}}+\bar{C}_{\mathrm{B}}}=\phi_{\mathrm{A}}+\phi_{\mathrm{B}},
$$

where the contribution of each ion is represented as follows:

$$
\phi_{\mathrm{A}}=\frac{C_{\mathrm{A}}(R)}{\bar{C}_{\mathrm{A}}+\bar{C}_{\mathrm{B}}}=\frac{f_{\mathrm{A}} \bar{C}_{\mathrm{A}}}{\bar{C}_{\mathrm{A}}+\bar{C}_{\mathrm{B}}}, \quad \phi_{\mathrm{B}}=\frac{C_{\mathrm{B}}(R)}{\bar{C}_{\mathrm{A}}+\bar{C}_{\mathrm{B}}}=\frac{f_{\mathrm{B}} \bar{C}_{\mathrm{B}}}{\bar{C}_{\mathrm{A}}+\bar{C}_{\mathrm{B}}}
$$

The temperature applied in the calculations is $25^{\circ} \mathrm{C}$, and the Bjerrum length is taken as $7.135 \AA$ in all cases.

When the integrated fraction of the counterion charge from inner boundary to position $r, Q(r)$, is given as

$$
Q(r)=w_{\mathrm{A}} q_{\mathrm{A}}(r)+w_{\mathrm{B}} q_{\mathrm{B}}(r),
$$

then the error of the electroneutrality in the $\mathrm{PB}$ solution is defined as the absolute difference of the value of cell boundary from unity, $\left|1-Q^{\mathrm{PB}}(R)\right|$. This quantity is utilized as one of the indexes of accuracy of the numerical PB calculation.

In the present system of $z_{\mathrm{A}}=z_{\mathrm{B}}=z$, the PB Eqs. (1)-(3) signify that the $\mathrm{PB}$ solution gives the identical free fraction and selectivity coefficient as a function of product of counterion valence $z$ and polyion charge density parameter $\xi$, whenever the cell dimensions, containing ion sizes, and ion composition are the same. Then, the comparisons of the MC results with the PB solutions are performed at the same $z \xi$ value for different $z$. Furthermore, the value $z^{2} C(r)$ must be identical in the case of different $z$ when other parameters are the same in the PB system.

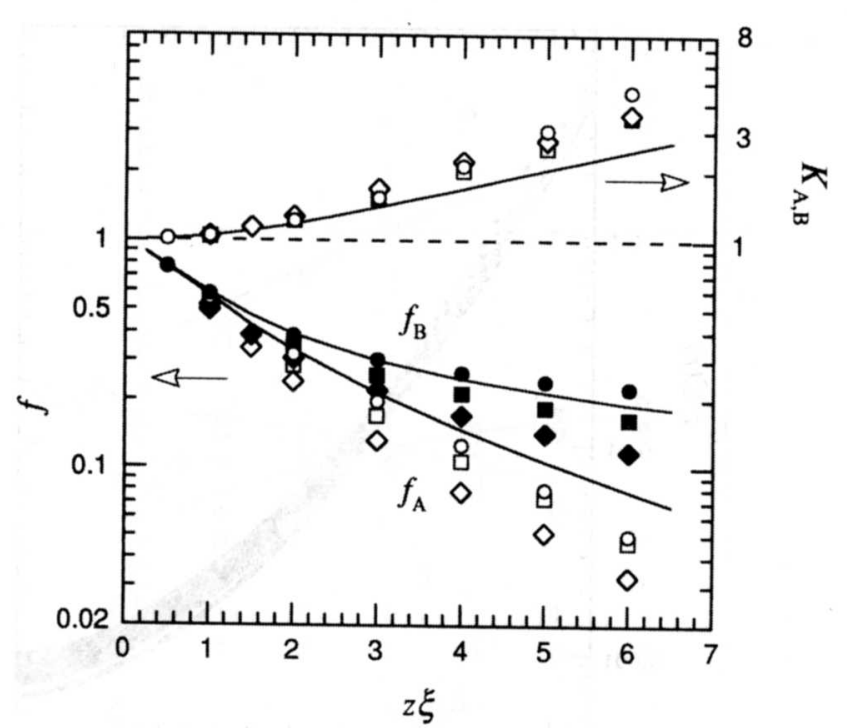

FIG. 1. Dependence of the free fractions $f_{\mathrm{A}}, f_{\mathrm{B}}$ (lower data, left axis) and of the selectivity coefficient $K_{\mathrm{A}, \mathrm{B}}$ (upper data, right axis) on the product of counterion valence $z$ and polyion charge density parameter $\xi$ in the mixture of two counterion species with different size $\left(\sigma_{\mathrm{A}}=3.0 \AA, \sigma_{\mathrm{B}}=5.0 \AA\right)$ and same valence $\left(z_{\mathrm{A}}=z_{\mathrm{B}}=z\right)$ at same number of two species of ion ( $w_{\mathrm{A}}$ $=0.5$ ). The $\mathrm{MC}$ results for $z=1$ (circles), 2 (squares), and 3 (diamonds) are presented together with the PB solution (lines) in the case of $a=5.0 \AA$ and $R=172.0 \AA$. Lower open symbols, $f_{\mathrm{A}}$; closed symbols, $f_{\mathrm{B}}$; and upper open symbols, $K_{\mathrm{A}, \mathrm{B}}$. Dashed line represents $K_{\mathrm{A}, \mathrm{B}}=1$.

\section{RESULTS}

\section{A. Dependence on charge density parameter}

First, the dependences on the charge density of the polyion are presented for the mixtures of two counterion species with the same valence $z$ and with different size $\left(\sigma_{\mathrm{A}}<\sigma_{\mathrm{B}}\right)$. As described above, if the dimensions of the cell and ions, and the ion composition are the same, the $\mathrm{PB}$ results are identical with the product of $z^{2}$ and the ion distribution $C(r)$ as a function of $z \xi$. In short, the values $f_{\mathrm{A}}, f_{\mathrm{B}}$, and $K_{\mathrm{A}, \mathrm{B}}$ in the same cell are common functions of $z \xi$ on the framework of the PB equation. On the other hand, the differences with the valence $z$ are expected due to the ion-ion correlation and the ion size effect in the MC simulations.

In Fig. 1 the MC results for $z=1,2$, and 3 are presented together with the PB solution in the case of $a=5.0 \AA, R$ $=172.0 \AA, \sigma_{\mathrm{A}}=3.0 \AA$, and $\sigma_{\mathrm{B}}=5.0 \AA$ at $w_{\mathrm{A}}=0.5$, corresponding to $C_{P}=2.5041 \times 10^{-3} \mathrm{~mol}$ equivalent $/ \mathrm{dm}^{3}$ at $\xi$ $=1$. As shown in Eq. (7), $C_{P}$ is proportional to $\xi$.

When $z \xi$ is less than unity, the selectivity coefficient due to the difference of the ion radius is not remarkable for any $z$. With high value $z \xi$ exceeding unity, the free fractions of both ions are separated, and the selectivity apparently rises.

The deviations between the MC result and the PB calculation become significant in still higher $z \xi$ range. The free fractions of the higher valence counterion apparently decrease downward in comparison with the PB solutions. However, the selectivity coefficient is not so sensitive as the free fractions. In the range $z \xi<2$, the selectivity coefficients from the MC simulations are indistinguishable within the statistical error. At $z \xi=2$, the order of selectivity is the same as order of valence. The monovalent ion distribution is very 

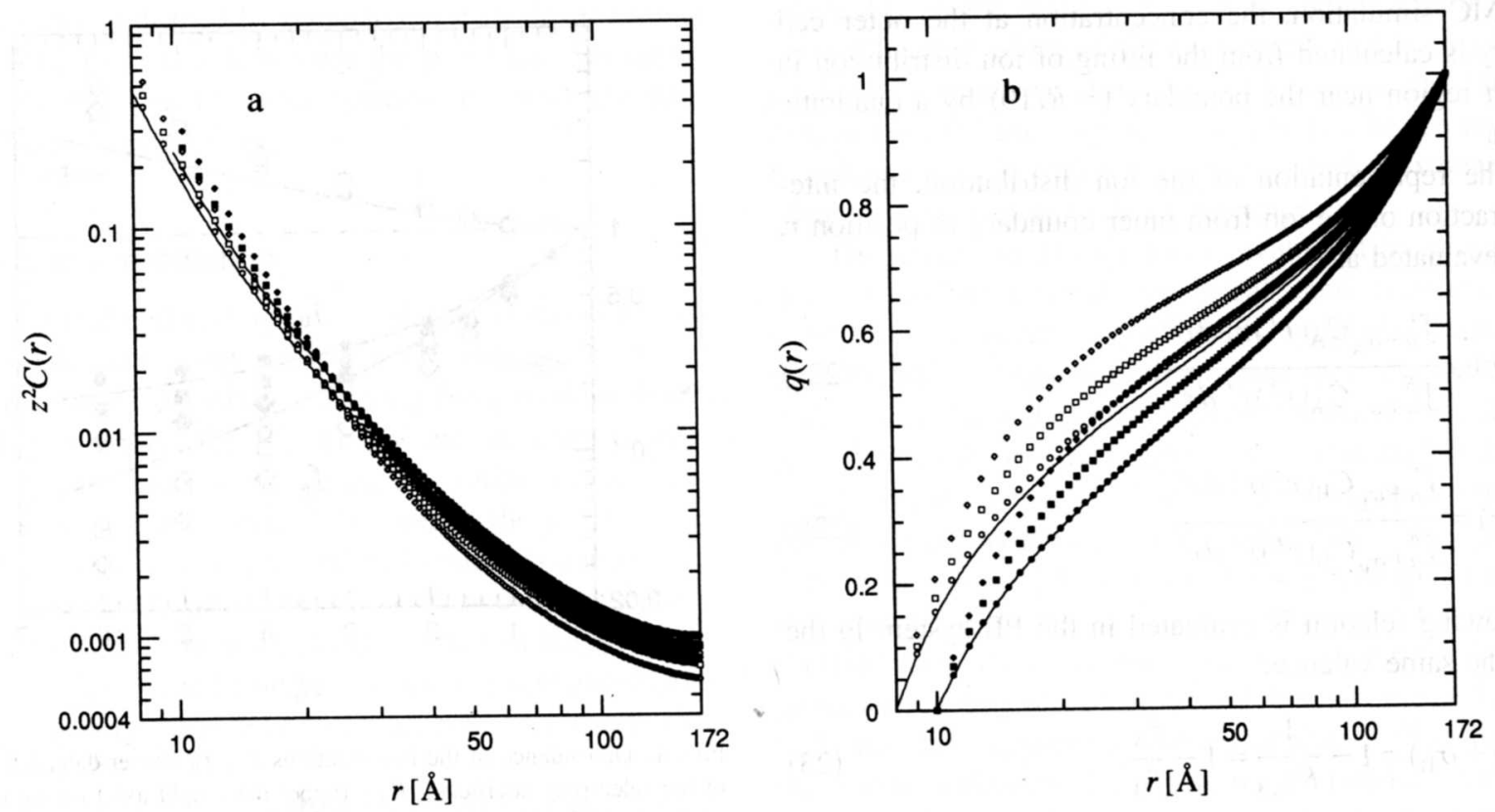

FIG 2 Comparison of the ion distributions $z^{2} C(r)$ (a) (log-log plot) and (b) of the integrated fractions $q(r)$ of both counterions at $z \xi=2$ in Fig. 1. The MC results for $z=1 \quad(\xi=2$, circles), 2 ( $\xi=1$, squares), and 3 ( $\xi=2 / 3$, diamonds) are presented together with the PB solutions (lines). Open symbols, ion $A$ $\left(\sigma_{\mathrm{A}}=3.0 \AA\right)$; closed symbols, ion $\mathrm{B}\left(\sigma_{\mathrm{B}}=5.0 \AA\right)$. Other parameters are the same as in Fig. 1 .

close to the PB solutions (see Fig. 2). The selectivity in the trivalent ions is also slightly larger than the divalent ions even in still higher $z \xi$ range.

In the mixed monovalent counterion systems, extraordinary behavior appears in high $z \xi(\geqslant 3)$ range. The free fraction of the large ion $f_{\mathrm{B}}$ is higher than the $\mathrm{PB}$ solution, and the free fraction of the small ion $f_{\mathrm{A}}$ goes downward. In the range $z \xi \geqslant 5$, higher selectivities are observed even in comparison with the case of the trivalent ions. This anomaly in the monovalent ions is obviously due to very high accumulation of the ions with finite ion size near the polymer rod surface.

The corresponding forms of the ion distribution $z^{2} C(r)$ and the integrated fractions of each ion at $z \xi=2$ and 5 in Fig. 1 are shown in Figs. 2 and 3, respectively. The accumulation of the multivalent counterions in the vicinity of the polyion is higher in the MC results than the $\mathrm{PB}$ solution considering the Coulombic interaction only. This deviation
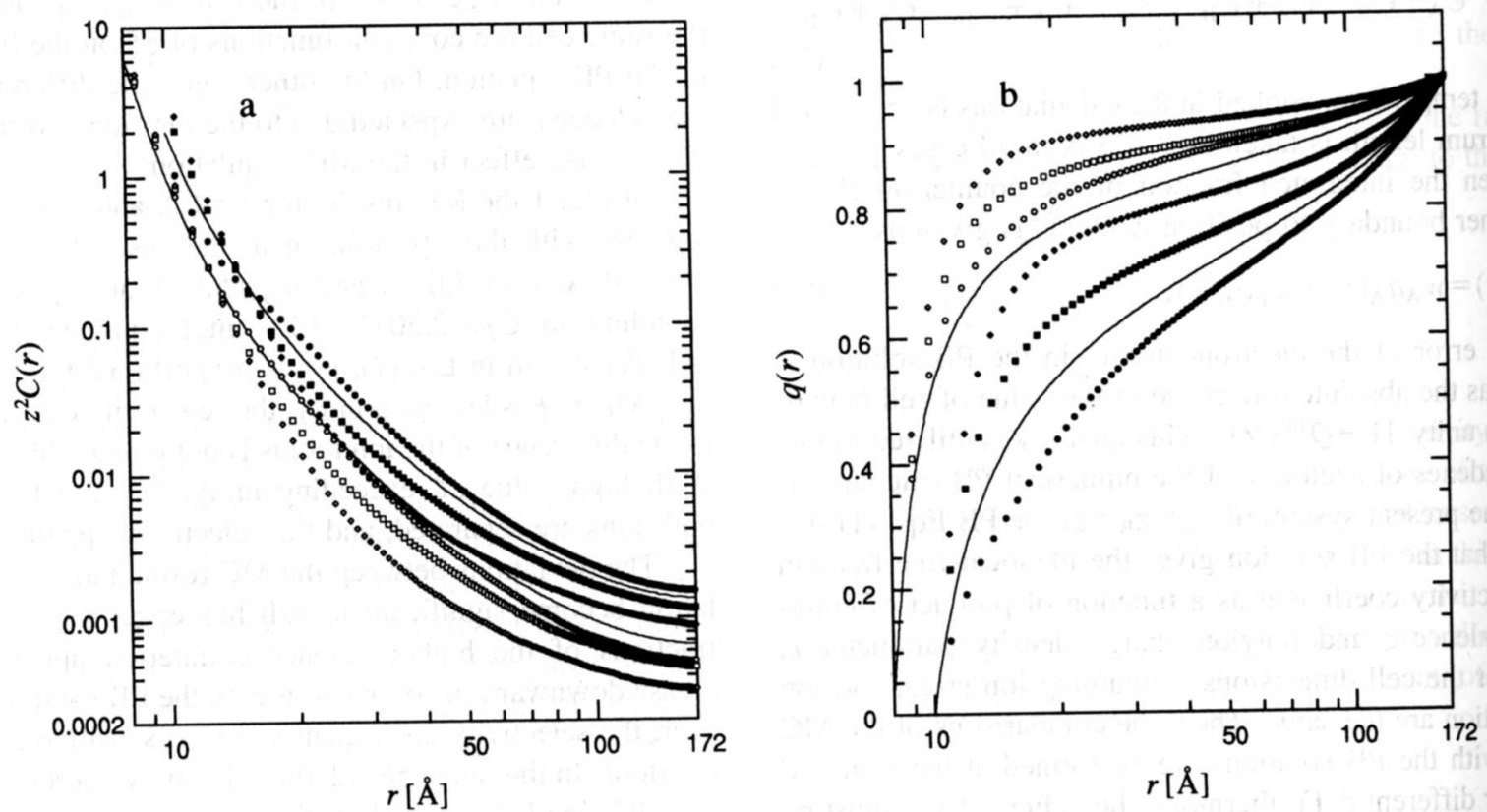

FIG. 3. Comparison of the ion distributions, (a) $z^{2} C(r)$ and (b) of the integrated fractions, $q(r)$ of both counterions at $z \xi=5$ in Fig. 1 . The MC results for $z=1(\xi=5), 2(\xi=2.5)$, and $3(\xi=5 / 3)$ are presented together with the PB solutions. Other parameters and details are the same as in Fig. 1 and 2. 
of the MC from the PB result is significant at the high charge density. This seems to be an appearance of the ion-ion correlations between individual ions ignored in the PB system.

In the MC result for the monovalent ions in Fig. 3(a) shoulders of the distribution are apparent for both ions. A part of small ion A is strongly accumulated within the first "layer" close to the polyion rod, and the remaining ions are pushed away by the repulsion of the core potential. Then, the irregularity of the ion distribution appears. In particular, the large ions B are repelled far from the polyion, and show a shoulder of the ion distributions in the second layer (at $r$ $\left.\approx a+2 \sigma_{\mathrm{A}}+\sigma_{\mathrm{B}}\right)$. As a result, high free fraction of ion $\mathrm{B}$ and low free fraction of ion $A$ are observed. The feature for monovalent counterion completely disappeared at $z \xi=2$ in the same cell dimensions as shown in Fig. 2. The PB equation seems to be a good approximation for the monovalent counterions under these conditions.

\section{B. Dependence on counterion size and polymer radius}

In the PB system, the free fractions and the selectivity coefficient are determined by the ratios of $\left(a+\sigma_{\mathrm{B}}\right)$ to $(a$ $+\sigma_{\mathrm{A}}$ ) and to $R$ at given $z \xi$ and $w_{\mathrm{A}}$. As described below, the dependence of the selectivity coefficient on $R$ is not so remarkable. The selectivity depends mainly on the ratio ( $a$ $\left.+\sigma_{\mathrm{B}}\right) /\left(a+\sigma_{\mathrm{A}}\right)$ in the PB system unless $R$ is small. However, even if the ratio is fixed, various combinations are possible for the mobile ion size and the polyion radius in the MC system. When many bulky ions are contained, the appearance of the ion size effect becomes significant. The selectivity is expanded in the MC simulations, if the difference in the radii of counterion species is large.

When the radius of ion $\mathrm{A} \sigma_{\mathrm{A}}$ is fixed at $3.0 \AA$, the dependence of each free fraction $f_{\mathrm{A}}, f_{\mathrm{B}}$ and the selectivity coefficient $K_{\mathrm{A}, \mathrm{B}}$ on the radius of ion B $\sigma_{\mathrm{B}}$ are shown for monovalent and divalent ion mixtures at $z \xi=5$ and $w_{\mathrm{A}}$ $=0.5$ in Fig. 4 . The other parameters of the polyion and cell are the same in Fig. 1. The high selectivity for the small ion is represented when the difference of the ion radius is large. At lower $z \xi$, the dependence of the selectivity coefficient on the radius of ion is weaker.

In the mixture of divalent counterions, the free fraction from the MC simulation is always smaller than that of the PB solution. This means that the MC result of the concentration near the polyion is larger than the PB result. The MC result for the larger ion is closer to that of $\mathrm{PB}$ result than that for the smaller one. The dependence of the selectivity coefficient on the ion size by the MC simulation is greater than that of the PB solution.

When the ion $\mathrm{B}$ is bulky in high $z \xi$ range, an extraordinary ion size effect appears in the case of the monovalent counterions. For large $\sigma_{\mathrm{B}}$, the considerably large deviations of the selectivity coefficient between the $\mathrm{MC}$ and the $\mathrm{PB}$ methods are observed as shown in Fig. 4. At $\sigma_{\mathrm{B}}=6 \AA$, an extensive shoulder appears in the distribution of ion $\mathrm{B}$ of the MC result. If the radii of both ions are small $(\leqslant 3 \AA)$, the extraordinary effect of ion size vanishes. Similar tendencies of the ion binding selectivity were observed and analyzed in the simulation for the monovalent cation competition to

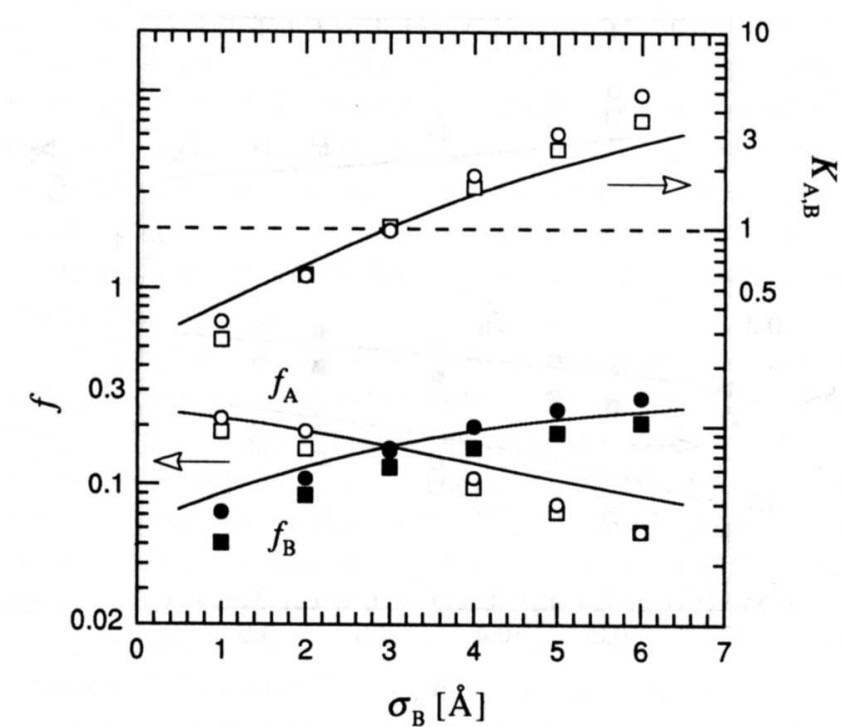

FIG. 4. Dependence of the free fractions $f_{\mathrm{A}}, f_{\mathrm{B}}$ and of the selectivity coefficient $K_{\mathrm{A}, \mathrm{B}}$ on the radius of ion B $\sigma_{\mathrm{B}}$ in the mixture of two counterion species with fixed radius of ion $\mathrm{A} \sigma_{\mathrm{A}}=3 \AA$ at $a=5 \AA, z \xi=5, R=172.0 \AA$, and $w_{A}=0.5$. The $\mathrm{MC}$ results for $z=1$ (circles) and 2 (squares) are presented together with the PB solutions (lines). Other details are the same as in Fig. 1.

DNA. ${ }^{34,35}$ At lower $z \xi$, the free fractions of the MC results are very close the $\mathrm{PB}$ solutions, and the dependence on the ion radius is smaller than that for divalent ions (data not shown).

The dependence on the polyion radius $a$ is shown at $z \xi$ $=4$ in Fig. 5, when the counterion radii are fixed. With decrease of the rod radius, the free fraction of small ion $\mathrm{A}$

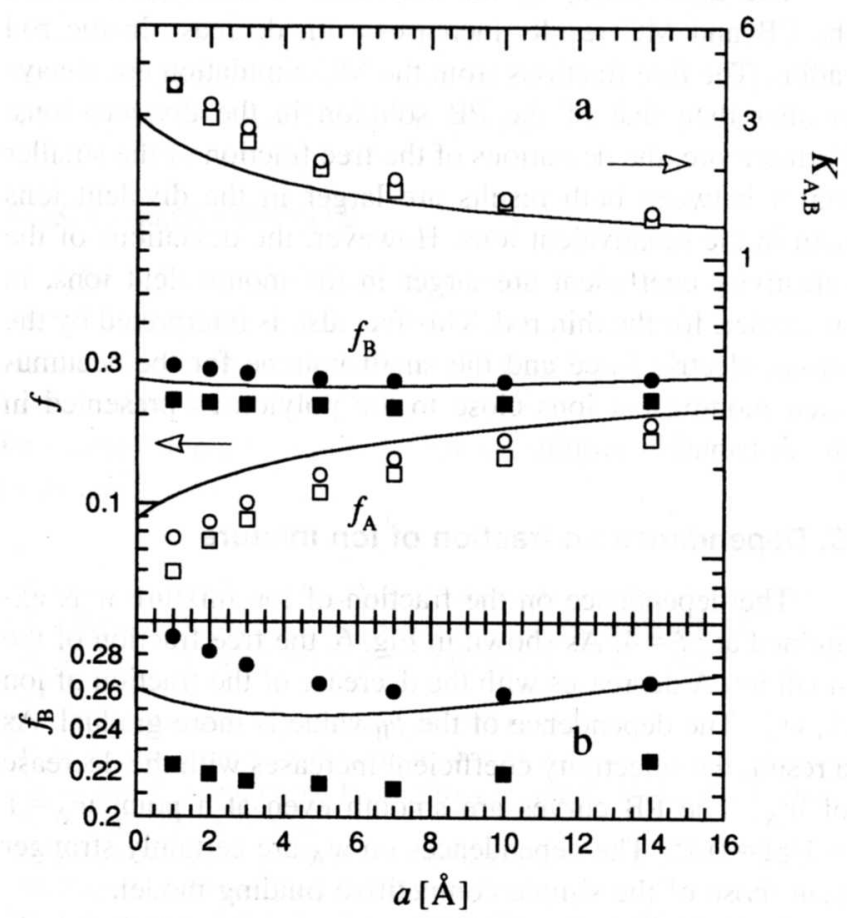

FIG. 5. Dependence of the free fractions $f_{\mathrm{A}}, f_{\mathrm{B}}$ and of the selectivity coefficient $K_{\mathrm{A} . \mathrm{B}}$ on the radius of the polyion rod $a$ in the case of the fixed ion radii $\sigma_{\mathrm{A}}=3 \AA$ and $\sigma_{\mathrm{B}}=5 \AA$ at $z \xi=4, R=172.0 \AA$ and $w_{A}=0.5$ (a). (b) expanded plot for $f_{\mathrm{B}}$. Other details are the same as in Fig. 1. 


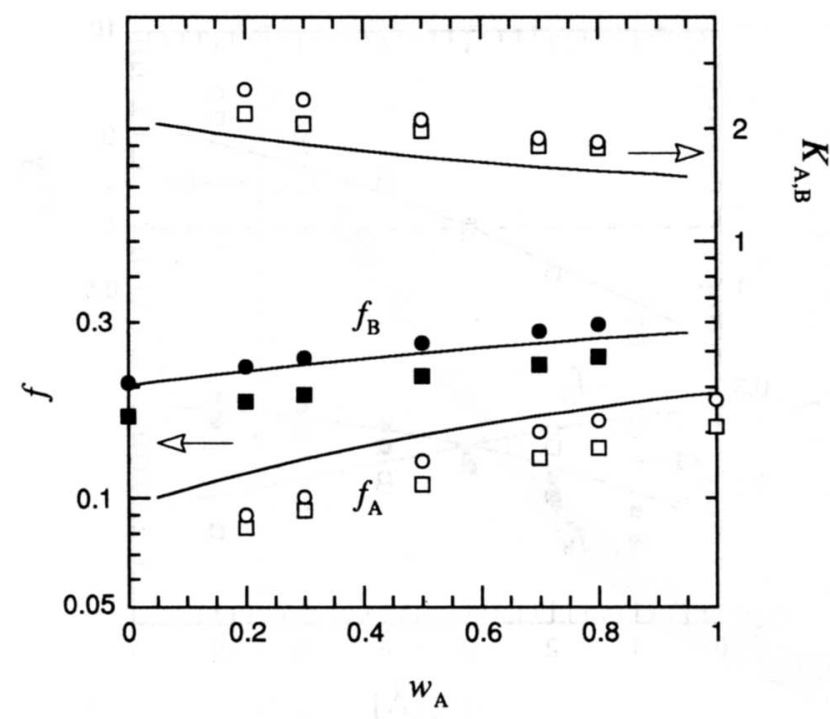

FIG. 6. Dependence of the free fractions $f_{\mathrm{A}}, f_{\mathrm{B}}$ and of the selectivity coefficient $K_{\mathrm{A} . \mathrm{B}}$ on the fraction of ion mixture $w_{\mathrm{A}}$ in the case of the fixed radii $a=5 \AA, \sigma_{\mathrm{A}}=3 \AA$, and $\sigma_{\mathrm{B}}=5 \AA$ at $z \xi=4$ and $R=172.0 \AA$. Other details are the same as in Fig. 1 .

decreases considerably. The free fraction of large ion B is not affected so. Then, the selectivity coefficient increases with thinning the rod in all cases. Notably, the free fraction of large ion $\mathrm{B}$ has a minimum around $a \approx 4.6 \AA$ by the $\mathrm{PB}$ calculation. The minimum point must exist in the MC simulations, and its position seems to be greater than that of the PB result [Fig. 5(b)]. However, it is difficult to determine the point accurately from the MC results due to the statistical error. This indicates that the accumulated small ions around a very thin rod push out the large ions.

The discrepancy of the selectivity coefficient between the PB and MC results increases with decrease in the rod radius. The free fractions from the $\mathrm{MC}$ simulation are always smaller than that of the PB solution in the divalent ions. Furthermore, the deviations of the free fraction of the smaller ion A between both results are larger in the divalent ions than in the monovalent ions. However, the deviations of the selectivity coefficient are larger in the monovalent ions, in particular, for the thin rod. This fact also is interpreted by the strong electric force and the small volume for the accumulated monovalent ions close to the polyion as presented in the distribution profile.

\section{Dependence on fraction of ion mixture}

The dependence on the fraction of ion mixture $w$ is examined at $z \xi=4$. As shown in Fig. 6, the free fraction of the small ion A decreases with the decrease of the fraction of ion $\mathrm{A}, w_{\mathrm{A}}$. The dependence of the $f_{\mathrm{B}}$ value is more gradual. As a result, the selectivity coefficient increases with the decrease of $w_{\mathrm{A}}$. The PB curves are smooth even at a point $w_{\mathrm{A}}=1$ $-1 / z \xi=0.75$. The dependences on $w_{\mathrm{A}}$ are certainly stronger than those of the simple competitive binding model.

In the $\mathrm{MC}$ results, small ions $\mathrm{A}$ are accumulated strongly around the polyion with lowering $w_{\mathrm{A}}$. This tendency is emphasized in the case of monovalent ions due to the size effect. The influence is exhibited as a higher selectivity coef-

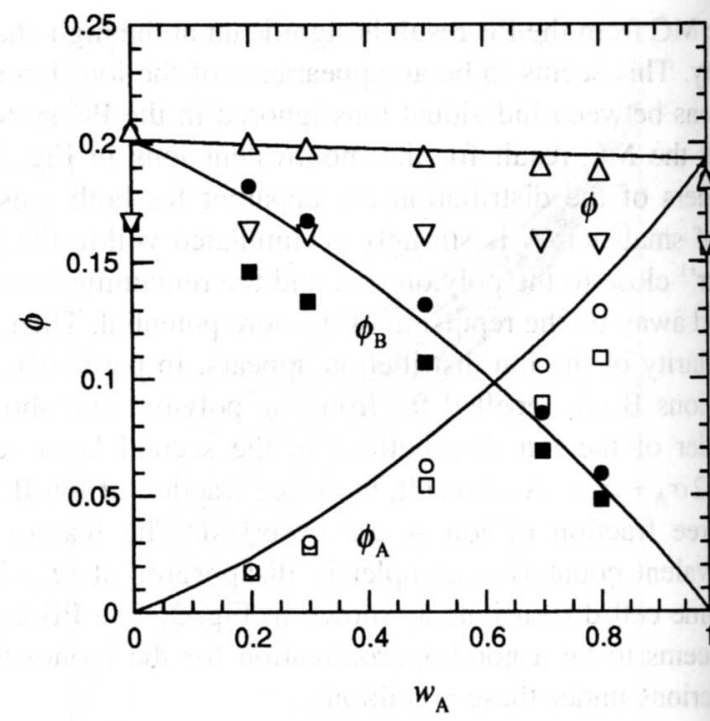

FIG. 7. Osmotic coefficient $\phi$ and its fraction of each ion $\phi_{\mathrm{A}}, \phi_{\mathrm{B}}$ depending on the fraction of ion mixture $w_{\mathrm{A}}$ under the same conditions in Fig. 6 . The MC results for $z=1\left(\phi_{\mathrm{A}}\right.$, open circles; $\phi_{\mathrm{B}}$, closed circles; and $\phi_{\text {, }}$ triangles) and 2 ( $\phi_{\mathrm{A}}$, open squares; $\phi_{\mathrm{B}}$, closed squares; and $\phi$, inverted triangles) are presented together with the PB solutions (lines).

ficient in the low $w_{\mathrm{A}}$ range. For the divalent ion mixture, the dependences on $w_{\mathrm{A}}$ of the MC results are almost parallel to the PB calculations in the log plot.

In Fig. 7, the osmotic coefficient, $\phi$, and the contribution of each ion are shown from the calculations in Fig. 6. In the figure of the linear plot, the deviations between the MC and $\mathrm{PB}$ results look very large in the divalent ions. This deviation in the multivalent ions decreases with decrease of the poly. ion charge density. The MC results for monovalent ions are close to the PB results in spite of the size effect. The deviations of contributions from both ions cancel each other in the total value $\phi$. Then, this value is not so sensitive to the fraction of mixture.

\section{Dependence on polymer concentration}

When other parameters are the same, the dependence on cell radius $R$ is investigated to check the polymer concentration dependence. From Eq. (7), the radius $R$ dependence can be represented as a dependence on the value $C_{P} / \xi$. This dependence is shown at $\sigma_{\mathrm{A}}=3.0 \AA, \sigma_{\mathrm{B}}=5.0 \AA$, and $z \xi=4$ in Fig. 8. The free fractions of both ions decrease with the dilution of the solution. The selectivity coefficient decreases gradually with the dilution as a result.

The MC results in the monovalent ions are almost parallel to the PB ones. On the other hand, the deviation of the MC from the PB result in the divalent ions increases with increase in the polymer concentration. This suggests the stronger effect of the ion-ion correlation between the divalent ions. However, the selectivity coefficient is insensitive to the difference.

By the extrapolation of $C_{P} / \xi \rightarrow 0(R \rightarrow \infty)$, the value by the $\mathrm{PB}$ method tends to $K_{\mathrm{A}, \mathrm{B}}=1.64$ in the limit of infinite dilution. The limits of the MC results seem to be slightly larger than this value. Nevertheless, an apparent difference cannot be determined by taking account of the statisti- 


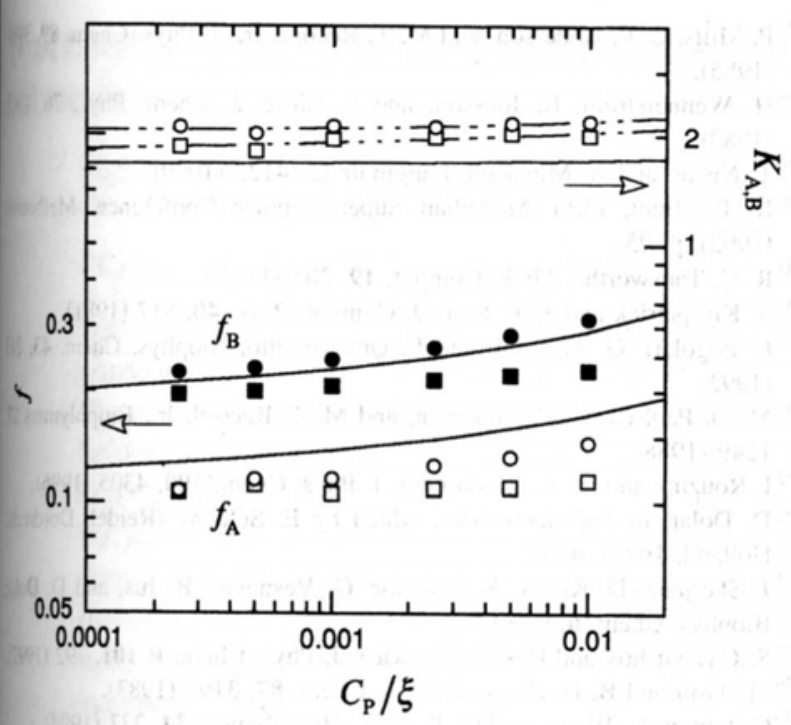

AG. 8. Dependence of the free fractions $f_{\mathrm{A}}, f_{\mathrm{B}}$ and of the selectivity wefficient $K_{\mathrm{A}, \mathrm{B}}$ on the value $C_{\mathrm{P}} / \xi\left[\mathrm{mol}\right.$ equivalent $\left./ \mathrm{dm}^{3}\right]$ in the case of the tred radii $a=5 \AA, \sigma_{\mathrm{A}}=3 \AA$, and $\sigma_{\mathrm{B}}=5 \AA$ at $z \xi=4$ and $w_{\mathrm{A}}=0.5$. The wh-dotted lines denote the least-square fittings of the MC data of the sectivity coefficient by a function $K_{\mathrm{A}, \mathrm{B}}=K_{\mathrm{A}, \mathrm{B}}^{0}+\alpha\left(C_{P} / \xi\right)^{\beta}$, where $K_{\mathrm{A}, \mathrm{B}}^{0}$ is Iliniting value of $K_{\mathrm{A}, \mathrm{B}}$ in the infinite dilution; $\alpha$ and $\beta$, fitting parameters. Other details are the same as in Fig. 1.

al error. These indicate that the finite fraction of ion $\mathrm{A}$ remains in the inner region $\left(a+\sigma_{\mathrm{A}} \leqslant r \leqslant a+\sigma_{\mathrm{B}}\right)$ even in the infinite dilution [see Eq. (23)]. In the range of $z \xi<1$, the $\mathbb{R}_{A B}$ value seems to tend to unity in both of the PB and MC results. These facts are consistent with the explanation of the counterion condensation hypothesis.

\section{N. DISCUSSION AND CONCLUSION}

In the mixture of the counterions with same valence, the size dependences of the free fraction and the selectivity coefficient on the various parameters are represented in detail. In the MC simulations, the degree of counterion accumulation is greater than that of the PB solutions in most cases. This means that the repulsive interactions between counterions near polyion are weak in the MC system in comparison with the mean-field approximation. The influence of the ionin correlation tends to accumulate the counterions near the polyion. The tendency of the counterion accumulation increses with increasing the valence of the counterion and the charge density of the polyion. The finite size of the ion partly ancels this influence. The weak repulsive interactions between counterions are strengthened by the core potential of the finite sized ions. Then, the MC and PB results in the monovalent ions are quite similar when the ion radii are in te range of 2-4 $\AA$ and the charge density is not so high. In general, the selectivity of small ion against large ion is emphasized for the multivalent ions in the MC simulations.

In the MC system for the monovalent counterion at high plyion charge density, the effect of core potential emerges significantly as a shoulder in the ion distribution, since very tigh ion density is induced by the electrostatic attraction to the polyion. Roughly estimating, when the ion concentration ecceds $10 \%$ of the close-packed concentration around the polyion rod, the extraordinary size effect appears due to the frequent collisions. Under this condition, there are some cases where the second peak in the distribution is observed as a result of the growth in the irregularity. This extraordinary effect of the ion size can also be represented in the system containing single counterion species. It is clearly exhibited in the ion selectivity depending on the difference of ion radius.

If the polyion charge density is the same, the dependences on the parameters are larger in the divalent ions than that in the monovalent ions, because the value $z \xi$ differs twofold. Furthermore, the deviations of the MC results from the PB solutions in the multivalent ions are greater than those in the monovalent ions for the parameters. This tendency is observed more clearly at higher charge density.

The present MC results indicate that the ion-ion correlations and the effect of the ion radius make the ion selectivity larger than that predicted by the PB calculations in high $z \xi$ range, in particular, for the multivalent counterion mixture. Furthermore, the pronounced size effect must appear on the condition that the concentration of the counterion is particularly high in the vicinity of the polyion. Reconsiderations on the interpretations of the experiments are required by taking these points into account. For example, it is expected that the effects found in the present MC simulations can clarify some differences between the experiment and the PB solution in the osmotic coefficient of the salt-free polyelectrolyte solution. ${ }^{36,37}$

Various effects are recognized as factors which modify the ion distribution, such as dielectric discontinuity, dielectric saturation, polymer configuration and conformation, specific site binding of the ions, and so on. ${ }^{38-41}$ The primitive model must not be enough for the reproduction of the experimental results without reservation due to its simplicity. It is believed, however, that the present approach is still valuable for the elucidation of the ion selectivity and other properties. A study on the mixed-valence systems is now in progress.

\section{ACKNOWLEDGMENTS}

The MC calculations in this study were executed on the vector processor in the information processing center of Hamamatsu University School of Medicine. The subroutines in Scientific Subroutine Library II (SSLII) by Fujitsu Limited were adopted for solving the ordinary differential equations and so on.

${ }^{1}$ F. Oosawa, Polyelectrolytes (Marcel Dekker, New York, 1971), Chap. 4.

${ }^{2}$ T. Alfrey, Jr., P. W. Berg, and H. Morawetz, J. Polym. Sci. 7, 543 (1951).

${ }^{3}$ R. M. Fuoss, A. Katchalsky, and S. Lifson, Proc. Natl. Acad. Sci. U.S.A. 37, 579 (1951).

${ }^{4}$ A. Katchalsky, Pure Appl. Chem. 26, 327 (1971).

${ }^{5}$ C. F. Anderson and M. T. Record, Jr., in Structure and Dynamics: Nucleic Acids and Proteins, edited by E. Clementi and R. H. Sarma (Adenine, New York, 1983), p. 301.

${ }^{6}$ V. A. Bloomfield and I. Rouzina, Methods Enzymol. 295, 364 (1998).

${ }^{7}$ M. Fixman, J. Chem. Phys. 70, 4995 (1979).

${ }^{8}$ R. J. Bacquet and P. J. Rossky, J. Phys. Chem. 92, 3604 (1988).

${ }^{9}$ T. Das, D. Bratko, L. B. Bhuiyan, and C. W. Outhwaite, J. Chem. Phys. 107, 9197 (1997).

${ }^{10}$ M. Le Bret and B. H. Zimm, Biopolymers 23, 271 (1984).

"V. Vlachy and A. D. J. Haymet, J. Chem. Phys. 84, 5874 (1986). 
${ }^{12}$ C. F. Anderson and M. T. Record, Jr., Annu. Rev. Biophys. Biophys. Chem. 19, 423 (1990).

${ }^{13}$ B. Jayaram and D. L. Beveridge, Annu. Rev. Biophys. Biomol. Struct. 25, 367 (1996).

${ }^{14}$ H. Ni, C. F. Anderson, and M. T. Record, Jr., J. Phys. Chem. B 103, 3489 (1999).

${ }^{15}$ G. R. Pack, L. Wong, and G. Lamm, Biopolymers 49, 575 (1999).

${ }^{16}$ N. Korolev, A. P. Lyubartsev, A. Rupprecht, and L. Nordenskiöld, Biophys. J. 77, 2736 (1999).

${ }^{17}$ N. Korolev, A. P. Lyubartsev, A. Rupprecht, and L. Nordenskiöld, J. Phys. Chem. B 103, 9008 (1999).

${ }^{18}$ D. Dolar and J. Skerjanc, J. Polym. Sci., Polym. Phys. Ed. 14, 1005 (1976).

${ }^{19}$ H. P. Gregor and J. M. Gregor, J. Chem. Phys. 66, 1934 (1977).

${ }^{20}$ D. Dolar and A. Peterlin, J. Chem. Phys. 50, 3011 (1969).

${ }^{21}$ G. Weisbuch and M. Guéron, J. Phys. Chem. 85, 517 (1981).

${ }^{22}$ G. S. Manning, J. Chem. Phys. 51, 924 (1969).

${ }^{23}$ D. Stiger, J. Colloid Interface Sci. 53, 296 (1975).

${ }^{24}$ J. H. Verner, SIAM (Soc. Ind. Appl. Math.) J. Numer. Anal. 15, 772 (1978).

${ }^{25} \mathrm{~N}$. Metropolis, A. W. Rosenbluth, M. N. Rosenbluth, A. H. Teller, and E. Teller, J. Chem. Phys. 21, 1087 (1953).

${ }^{26}$ T. Nishio, Biophys. Chem. 57, 261 (1996).
${ }^{27}$ P. Mills, C. F. Anderson, and M. T. Record, Jr., J. Phys. Chem. 89, 3994 (1985).

${ }^{28} \mathrm{H}$. Wennerström, B. Jönsson, and P. Linse, J. Chem. Phys. 76, 4665 (1982).

${ }^{29}$ T. Nishio and A. Minakata, Langmuir 15, 4123 (1999).

${ }^{30}$ R. P. Brent, Fifth Australian Supercomputer Conference, Melboune (1992), p. 95.

${ }^{31}$ R. C. Tausworthe, Math. Comput. 19, 201 (1965).

${ }^{32}$ S. Kirkpatrick and E. P. Stoll, J. Comput. Phys. 40, 517 (1981).

${ }^{33}$ F. Fogolari, G. Manzini, and F. Quadrifoglio, Biophys. Chem. 43, 21: (1992).

${ }^{34}$ M. D. Paulsen, C. F. Anderson, and M. T. Record, Jr., Biopolymers $\eta$, 1249 (1988).

${ }^{35}$ I. Rouzina and V. A. Bloomfield, J. Phys. Chem. 100, 4305 (1996).

${ }^{36}$ D. Dolar, in Polyelectrolytes, edited by E. Sélégny (Reidel, Dordrath, Holland, 1974), p. 97.

${ }^{37}$ J. Škerjanc, D. Kozak, S. Hočevar, G. Vesnaver, B. Jus, and D. Dolz, Biophys. Chem. 6, 9 (1977).

${ }^{38}$ S. Gavryushov and P. Zielenkiewicz, J. Phys. Chem. B 101, 792 (1997),

${ }^{39}$ M. Troll and B. H. Zimm, J. Phys. Chem. 87, 3197 (1983).

${ }^{40}$ G. Lamm, L. Wong, and G. R. Pack, Biopolymers 34, 227 (1994).

${ }^{41}$ M. Voué and C. Gillet, Biophys. Chem. 51, 9 (1994). 